\title{
Bronchial margins in lung cancer resection specimens: utility of frozen section and gross evaluation
}

\author{
Susan J Maygarden ${ }^{1,2}$, Frank C Detterbeck ${ }^{3}$ and William K Funkhouser ${ }^{1,2}$ \\ ${ }^{1}$ Department of Pathology; ${ }^{2}$ Department of Laboratory Medicine and ${ }^{3}$ Department of Cardiothoracic Surgery, \\ University of North Carolina at Chapel Hill, Chapel Hill, NC, USA
}

\begin{abstract}
Pathology reports for all lobectomy and pneumonectomy specimens at UNC Hospitals between 1991 and 2000 $(n=405)$ were reviewed for correlation between frozen section and final bronchial margin, gross distance between tumor and margin and tumor type. Frozen section was performed in 268 cases $(66 \%)$. A total of 243 were true negatives $(90.6 \%), 16(6.0 \%)$ were true positives, four $(1.5 \%)$ were false positives and five $(1.9 \%)$ were false negatives. The site of tumor in true-positive cases was mucosal (11), submucosal (three), lymphatics (one), peribronchial (one). The site of tumor in false-negative cases was submucosal (two), lymphatics (one), peribronchial (two). In 137 cases, no bronchial frozen section was performed; there was one case (0.7\%) with positive margin. There was no correlation between final margin positivity and distance between gross tumor and margin. Tumor distance to margin in positive margin cases varied from grossly involved to $3 \mathrm{~cm}$ away. There were 72 cases in which wedge resection was performed before lobectomy in which no gross tumor remained in the lobectomy, and in all cases final bronchial margins were negative. In all, 373 of cases (92\%) were nonsmall carcinomas. Of these, $10(2.7 \%)$ had positive margins. Tumors other than nonsmall cell carcinoma accounted for a disproportionate number of positive margins. In all, 3/6 of adenoid cystic/ mucoepidermoid carcinoma, 1/7 small cell carcinoma and 1/1 lymphoma cases had positive margins. In conclusion, frozen section evaluation of bronchial margins is helpful in central lung tumors. Mucosal tumor is preferentially identified in frozen section. Gross evaluation of margins is problematic, as intramucosal carcinoma or tumor in lymphatics may not be detected, but $3 \mathrm{~cm}$ was a 'safe' distance for gross tumor from margin. In lobectomies following wedge resection in which no gross tumor remained, all had negative margins. Salivary gland-type tumors have a high incidence of positive margins, and frozen section is particularly indicated in these tumors.

Modern Pathology (2004) 17, 1080-1086, advance online publication, 7 May 2004; doi:10.1038/modpathol.3800154
\end{abstract}

Keywords: lung cancer; bronchial; margins; surgical; resection

Optimal surgical treatment of lung carcinoma includes complete local resection of the primary tumor. To this end, resection margins should be uninvolved with tumor. Many surgical series have demonstrated adverse patient outcome with either microscopic or macroscopic residual tumor at the bronchial resection margin, ${ }^{1-13}$ and thus it is usually assumed that negative margins give the best chance for local control. However, poor outcomes with residual microscopic disease have not been reported by all authors. ${ }^{14-16}$

Correspondence: Dr SJ Maygarden, MD, Department of Pathology and Laboratory Medicine, CB 7525, University of North Carolina at Chapel Hill, Chapel Hill, NC 27599-7525, USA.

E-mail: susan.maygarden@pathology.unc.edu

Received 26 November 2003; revised 26 February 2004; accepted 28 February 2004; published online 7 May 2004
Surgical and pathologic methods vary to determine negative margins intraoperatively. Some authors recommend frozen sections of bronchial margins in all cases, ${ }^{4,17}$ while others recommend primarily gross evaluation with frozens reserved for cases in which tumor is within a defined distance of the margin. ${ }^{18,19}$ Distinctions have also been made about different prognosis for positive margins of mucosal bronchial carcinoma (in situ and/or invasive tumor involving mucosa or submucosa), extramucosal peribronchial invasive carcinoma, and carcinoma within lymphatics at the bronchial margin. ${ }^{4-7,9,11,17}$ In spite of the voluminous (and often conflicting) literature on this topic in the clinical cardiothoracic literature, little has been written in pathology journals about indications for frozen sections and gross evaluation of tumor distance to margins. This study was undertaken to 
look at the experience of bronchial margin evaluation at one institution, and to relate final margin status with gross distance from tumor, frozen section results and tumor type. Additionally, we evaluated whether type of operation (wedge biopsy followed by completion lobectomy or planned lobectomy/ pneumonectomy) influenced the incidence of positive bronchial margins.

\section{Materials and methods}

A computerized search of the surgical pathology files of all pulmonary lobectomy and pneumonectomy specimens at University of North Carolina Hospitals was done for the 10 year period 19912000. This yielded 405 cases, and the pathology reports of all cases were reviewed. The tumor type, gross distance between tumor and bronchial margin (if present in the report), results of frozen section of bronchial margin (if done) and status of final bronchial margins were tabulated for each case. A bronchial margin on either frozen section or final permanent section was considered positive if it showed invasive carcinoma or carcinoma in situ. Slides from all cases with either positive margins (at frozen section and/or at final case signout) or discrepancies between frozen and permanent margins were reviewed. Site of tumor in relation to the bronchus was tabulated in all positive cases.

Information about follow-up and subsequent clinical course was obtained from the computerized medical record for all positive cases.

\section{Results}

Pathologic data of the cases are summarized in Table 1. All patients had primary tumor excisions, none had preoperative chemotherapy or radiation therapy.

\section{Frozen Sections}

Frozen sections were carried out intraoperatively in 268 of the cases $(66 \%)$. The concordance rate between frozen and permanent diagnoses was $97 \%$, with 243 of the frozens being true negatives $(90.6 \%)$ and 16 being true positives $(6 \%)$. The site of the 16 true positive margins was mucosal in 11 cases, submucosal in three cases, within lymphatics in one case, and peribronchial in one case. In seven of the true positive cases the frozen section led to an extended surgical operation (additional lobectomy or bronchial sleeve resection performed), resulting in an ultimate negative margin. In one case of extensive adenoid cystic carcinoma extended surgery was attempted, but a negative margin was never obtained, and the patient died intraoperatively from massive hemorrhage (Figure 1). In the remaining
Table 1 Pathologic features of the 405 cases

\begin{tabular}{|c|c|}
\hline Type of carcinoma & No. of cases \\
\hline Squamous cell carcinoma & 175 \\
\hline Adenocarcinoma, NOS & 147 \\
\hline Adenosquamous & 15 \\
\hline Bronchoalveolar carcinoma & 11 \\
\hline ‘Non-small-cell carcinoma’ & 9 \\
\hline Large-cell undifferentiated carcinoma & 11 \\
\hline Small-cell carcinoma & 7 \\
\hline Mixed small- and large-cell carcinoma & 1 \\
\hline Basaloid carcinoma & 1 \\
\hline Sarcomatoid carcinoma & 3 \\
\hline Carcinoid tumor & 11 \\
\hline Atypical carcinoid tumor & 5 \\
\hline Large-cell neuroendocrine carcinoma & 1 \\
\hline Adenoid cystic carcinoma & 3 \\
\hline Mucoepidermoid carcinoma & 3 \\
\hline $\begin{array}{l}\text { Lymphoma (primary lung) mixed large and small } \\
\text { cleaved }\end{array}$ & 1 \\
\hline Melanoma (primary lung) & 1 \\
\hline \multicolumn{2}{|l|}{ Procedure } \\
\hline Wedge biopsy with completion lobectomy & 72 \\
\hline Lobectomy or pneumonectomy & 326 \\
\hline Lobectomy with bronchial sleeve resection & 7 \\
\hline \multicolumn{2}{|l|}{ Bronchial margin frozen section performed } \\
\hline No & 137 \\
\hline Final bronchial margin negative & 136 \\
\hline Final bronchial margin positive & 1 \\
\hline Yes & 268 \\
\hline Frozen section bronchial margin negative & $248^{\mathrm{a}}$ \\
\hline Frozen section bronchial margin positive & $20^{\mathrm{b}}$ \\
\hline Final bronchial margin negative & 254 \\
\hline Final bronchial margin positive & $14^{\mathrm{c}}$ \\
\hline
\end{tabular}

${ }^{a}$ The 248 negative frozen sections included 243 true negatives and five false negatives.

${ }^{\mathrm{b}}$ The 20 positive frozen sections included 16 true positives and four false positives.

${ }^{\mathrm{c}}$ Causes for these 14 positive bronchial margins are five false-negative frozen sections and nine cases in which additional surgery could not be done after frozen section was positive.

eight cases, additional surgery could not be performed, and these patients were referred for postoperative chemotherapy or radiation therapy.

There were four cases of false positives $(1.5 \%)$ : squamous metaplasia interpreted as dysplasia/carcinoma in situ, radiation changes interpreted as strongly suspicious for carcinoma and two cases of benign peribronchial lymphocytes interpreted as small cell carcinoma at frozen section (Figure 2). In both of these latter cases the primary tumor type (squamous cell carcinoma) was not known preoperatively. In all four cases the false positive diagnoses at frozen section did not change the surgery performed.

There were five cases of false negatives $(1.9 \%)$ : three sampling error (two cases of peribronchial invasive squamous cell carcinoma and one case with tumor in a lymphatic space, all of these were not present on the original frozen section) and two diagnostic errors (one missed adenocarcinoma and 

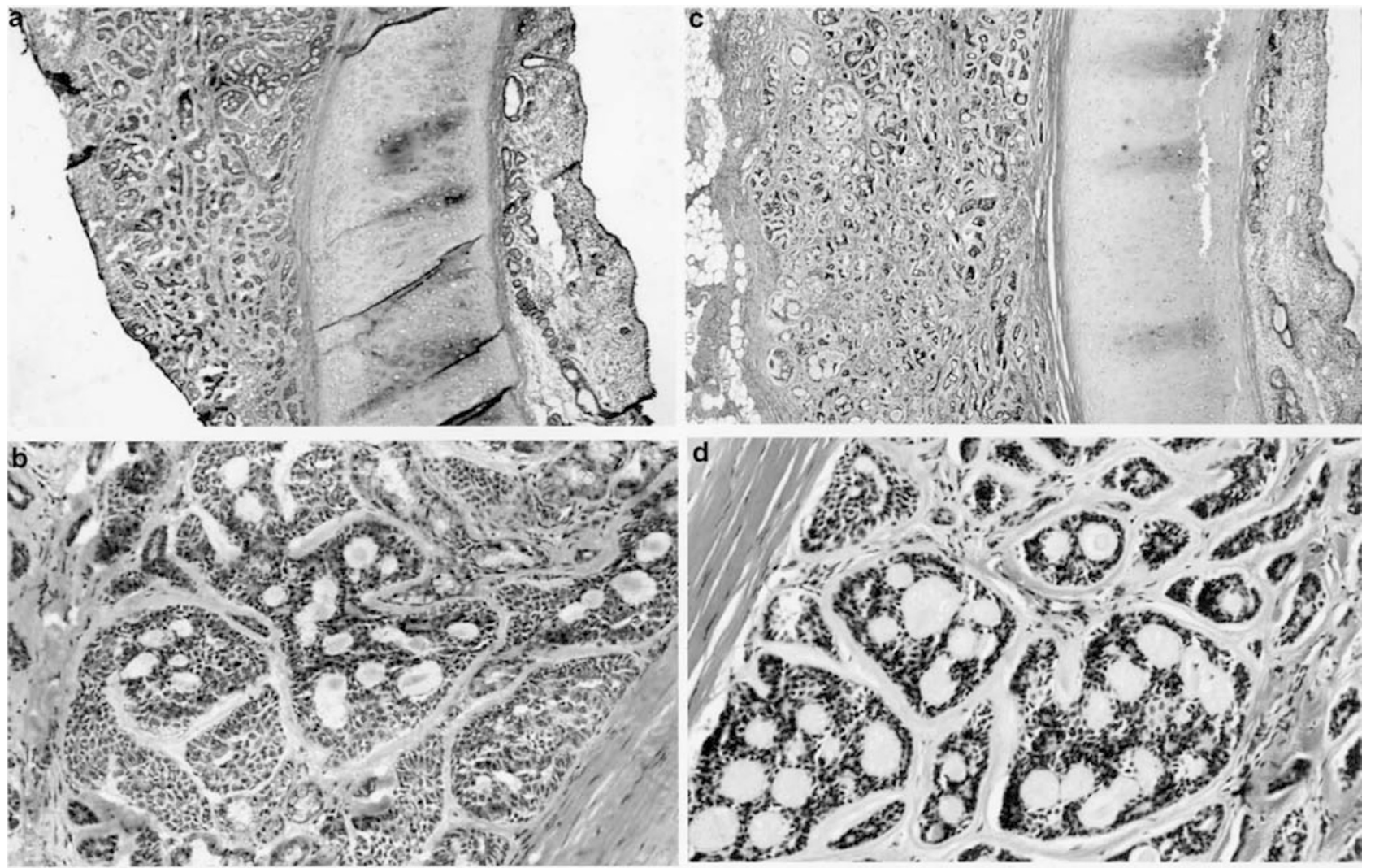

Figure 1 Adenoid cystic carcinoma with true-positive frozen section. (a) and (b) Frozen section showing sub-bronchial adenoid cystic carcinoma $(\mathrm{H} \& \mathrm{E}$, a: $\times 40, \mathbf{b}: \times 200)$. (c) and (d) Permanent sections showing nearly identical findings $(\mathrm{H} \& \mathrm{E}, \mathbf{c}: \times 40, \mathbf{d}: \times 200)$

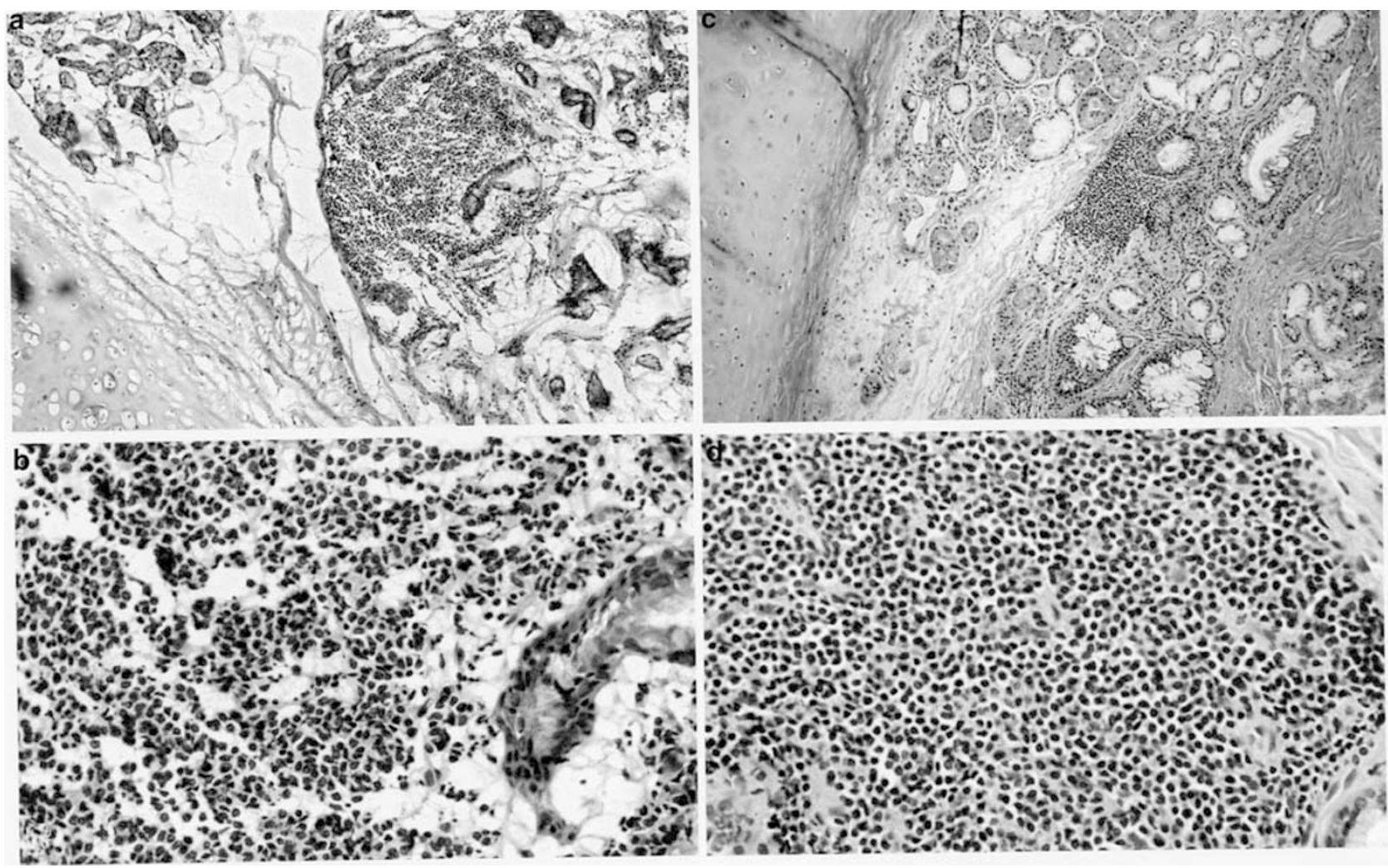

Figure 2 False-positive frozen section. Benign peribronchial lymphocytes interpreted as small-cell carcinoma on frozen section. (a) and (b) frozen section $(\mathrm{H} \& \mathrm{E}, \mathbf{a}: \times 100, \mathbf{b}: \times 400)$. (c) and (d) Permanent sections showing lymphocytes $(\mathrm{H} \& \mathrm{E}, \mathbf{a}: \times 100, \mathbf{b}: \times 400)$. 

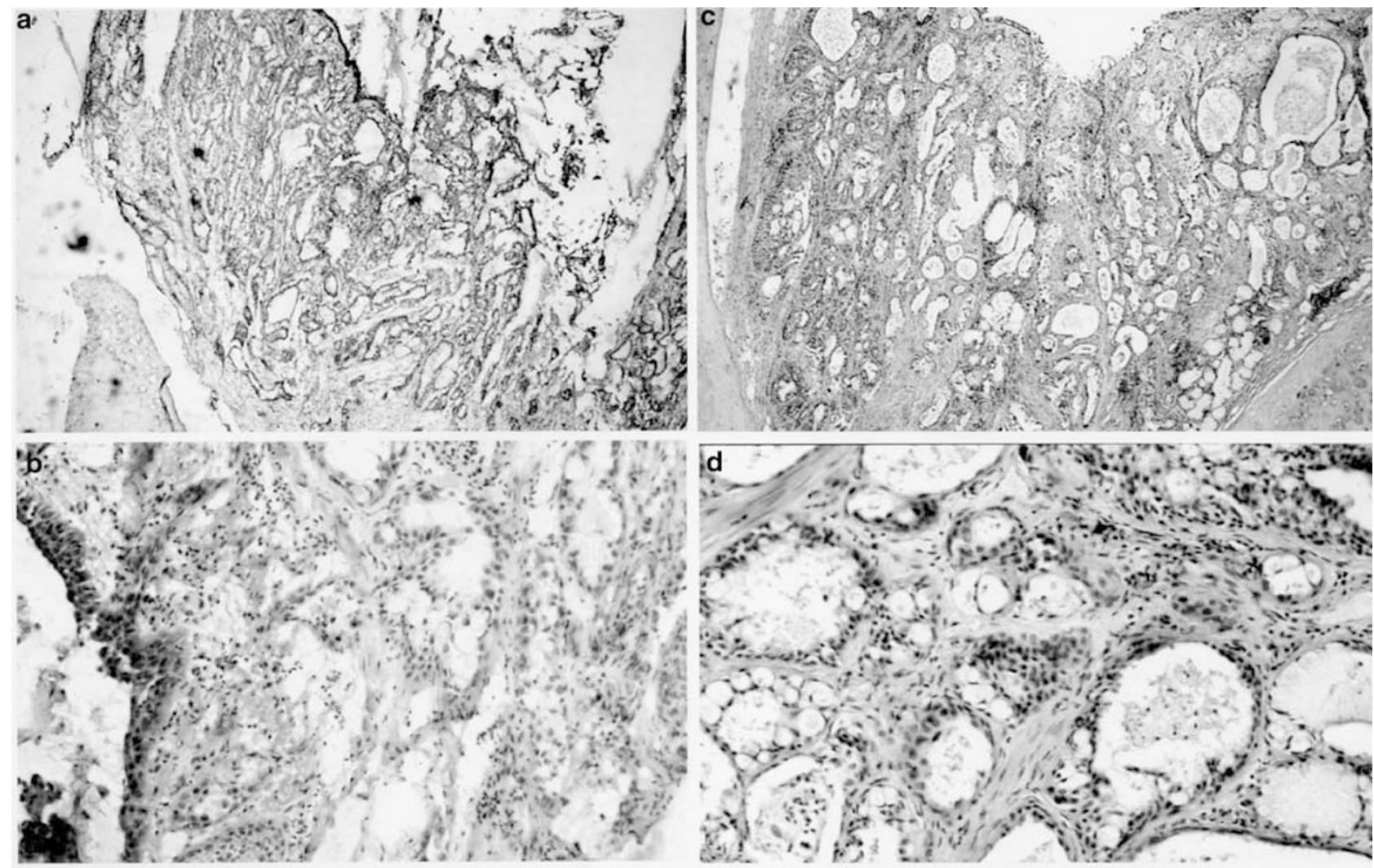

Figure 3 False-negative frozen section: Mucoepidermoid carcinoma interpreted as benign peribronchial mucinous glands on frozen section. (a) and (b) Frozen section (H\&E, a: $\times 40, \mathbf{b}: \times 200)$. (c) and (d) Permanent sections showing low-grade mucoepidermoid carcinoma $(\mathrm{H} \& \mathrm{E}, \mathbf{c}: \times 40, \mathbf{d}: \times 200)$.

one missed mucoepidermoid carcinoma (Figure 3), both submucosal). One of these patients (mucoepidermoid carcinoma) developed a local recurrence, had a completion pneumonectomy a year later and is currently free of tumor with a 9-year follow-up. Two patients (one with peribronchial squamous cell carcinoma, one with tumor in lymphatics) received postoperative radiation therapy but both developed local recurrences and one developed distant metastases. One patient (with adenocarcinoma) developed metastases in the contralateral lung. One patient (with peribronchial squamous cell carcinoma) developed superior vena cava syndrome and died perioperatively.

All frozen sections were interpreted by a board certified attending pathologist.

In 137 cases frozen sections were not performed. Of these there was one case $(0.7 \%)$ with a positive bronchial margin (mucosal tumor).

Analysis of the final margin status of all of the cases showed that there were 15 cases $(3.7 \%)$ with final margin positivity. The reasons for the 15 cases with ultimate final positive bronchial margins were inability to obtain a negative margin surgically when frozen section was positive in nine cases, falsenegative frozen section in five cases, and no frozen section requested in one case.

\section{Gross Evaluation}

Correlation between gross distance of tumor from margin and final margin positivity was problematic. Positive microscopic margins were found in cases that were up to $3 \mathrm{~cm}$ away from gross apparent tumor. Cases that had grossly negative but microscopically positive margins included examples of bronchial salivary gland-type tumors (adenoid cystic and mucoepidermoid carcinoma) and carcinoid tumor in which tumor undermined normal bronchial mucosa, carcinoma in situ extending proximally from an invasive tumor mass, tumor in peribronchial lymphatics, and lymphoma. Thus, gross evaluation alone is not adequate to ensure negative bronchial margins. Three centimetre was the maximum distance that microscopic tumor extended from a grossly visible tumor.

Conversely, there were cases in which the margin appeared grossly involved with tumor that were negative by frozen and permanent sections. Fibrous tissue and lymphoid tissue at the margin were the principal reasons for false-positive gross evaluation.

However, there were 72 cases in which a wedge resection was the first procedure performed, and after a frozen or permanent diagnosis of carcinoma was made a completion lobectomy was performed 
and there was no gross tumor in the lobectomy specimen. In all of these cases, there was a negative bronchial margin.

\section{Tumor Type}

The distribution of tumor types is shown in Table 1. The great majority of the tumors $(373,92 \%)$ were non-small-cell carcinoma (squamous cell carcinoma, adenocarcinoma and variants, large cell undifferentiated, and several unusual nonsmall cell tumors). Of $373(2.7 \%)$ of non-small-cell carcinoma, 10 had positive margins of invasive carcinoma. There were 16 carcinoid and atypical carcinoid tumors, seven small cell carcinomas, one mixed small cell and non-small-cell carcinoma, six salivary-gland tumors (three adenoid cystic carcinoma, three mucoepidermoid carcinoma), one primary lung lymphoma, and one primary lung melanoma. The salivary-gland-type tumors accounted for a disproportionate number of positive margins: two of three mucoepidermoid carcinomas and one of three adenoid cystic carcinomas had positive margins (overall $50 \%$ of the salivary-gland tumors). One of one case of lymphoma and one of seven cases of small-cell carcinomas also had positive margins.

\section{Discussion}

Residual tumor at a resection margin is divided into microscopic (R1) and macroscopic (R2) residual disease. ${ }^{20}$ Many but not all studies of positive margins of surgically treated lung cancers have shown adverse impact by residual disease. ${ }^{1-16}$

Microscopic residual tumor at the bronchial resection margin has then been divided into different patterns. Cotton ${ }^{1}$ first divided microscopic residual disease into mucosal tumor (spreading from a mucosal lesion), and extramucosal microscopic residual disease (tumor involving peribronchial soft tissues or tumor within lymphatics). ${ }^{1}$ Extramucosal tumor had a worse prognosis. Soorae divided microscopic residual disease into four patterns: direct submucosal extension of invasive cancer, carcinoma in situ, tumor present in lymphatics at the margin, and parabronchial invasive tumor. Submucosal and parabronchial lymphatic tumor had the worst prognosis. ${ }^{11}$ Snijder divided Stage I R1 non-small-cell cancers into carcinoma in situ, muscosal residual disease and peribronchial residual disease, and mucosal and peribronchial residual disease had statistically significant reduction in survival with no adverse effect for carcinoma in situ. ${ }^{10}$ Similar outcomes were found by Massard et $a l^{7}$ in a group of Stage I-III lung cancers, in which residual carcinoma in situ had no influence on survival, but peribronchial tumor had an adverse impact. Conversely, Ghiribelli divided residual microscopic diseases into mucosal and extramucosal tumor, but found no difference in survival between these patterns. ${ }^{4}$ Lymphatic space involvement at the bronchial margin has been shown to be an adverse prognostic factor in many studies. ${ }^{16,9,12}$

Gross evaluation of bronchial margins are known to be problematic. Tumors often have extensive microscopic proximal extension in excess of the apparent gross tumor. This is more common for central tumors $30.3 \%$ of the central tumors had microscopic tumor extending past the grossly visible tumor in one study. ${ }^{21}$ Peripheral tumors have been reported to have a lesser frequency of proximal extension $(18.9 \%)$, but a greater length of extension (15.7 vs $10.9 \mathrm{~mm}) .{ }^{21}$ Length and type of microscopic proximal extension has also been related to different tumor types. Squamous cell carcinoma shows a slightly greater rate of proximal extension than adenocarcinoma. When proximal extension is present, squamous cell carcinoma predominately shows mucosal spread, while adenocarcinoma shows peribronchial spread. Adenocarcinoma has also been shown to have an average longer proximal extension of tumor than squamous cell carcinoma. ${ }^{18}$ Suggested 'safe distances' for length of bronchial margins have ranged from 1.5 to $2 \mathrm{~cm} .^{1,18,19,22}$ Some authors have suggested taking longer margins for adenocarcinoma $(1.5 \mathrm{~cm}$ for squamous cell carcinoma, $2.0 \mathrm{~cm}$ for adenocarcinoma. ${ }^{22}$

Studies of lung cancer series report a wide range of positive margins, with rates of $1.1-17 \%$ of cases having residual tumor at the bronchial margin. ${ }^{4,11,17,19}$ Intraoperative FS examination of bronchial margins is suggested in most modern surgical series, but it is recognized that frozen sections can have false negative and positives. False-negative rates as high as $41.7 \%$ have been reported. ${ }^{6}$ Causes of false-negative frozens include sampling error and various reasons for interpretative error (mistaking invasive cancer for submucosal lymphocytes or submucosal glands, mistaking carcinoma in situ as squamous metaplasia, cautery artifact obscuring invasive carcinoma, etc). Some authors feel all lung cancer cases require intraoperative frozen section, ${ }^{4,17}$ while other suggest frozen section be reserved for cases that resection is planned within a defined distance from gross tumor. ${ }^{19}$

The impact of positive margins and the suggested response to this finding postsurgically vary among reports. As described above, outcomes vary with site of positive margins, with submucosal, peribronchial and lymphatic space involvement at margins found to be worse than mucosal positive margins in most studies. ${ }^{6,7,9,16,17,23}$ Several studies have found little benefit from postoperative radiation therapy., Chemotherapy added to radiation therapy may add additional benefit in some series. ${ }^{24}$

Bronchial salivary gland-type tumors, especially adenoid cystic carcinoma and mucoepidermoid carcinoma, are slow-growing neoplasms which originate in the bronchial glands. ${ }^{25-30}$ They are usually central, often submucosal, lung masses which extend locally, often through the bronchial 
wall and along the bronchial submucosa. ${ }^{29}$ Survival depends predominately on ability to obtain complete resection, tumor stage and grade. The great majority of mucoepidermoid carcinomas are of low grade, and patients with low-grade tumors in which negative margins can be obtained surgically can expect an excellent outcome. ${ }^{26,30}$ Hence, for this group, FS evaluation of surgical margins is a necessity. Since low-grade mucoepidermoid carcinoma often involves the bronchus only and not the underlying lung, lung-sparing surgery such as sleeve resections may provide local control without compromising lung function. ${ }^{12,26,31,32}$ High-grade mucoepidermoid carcinoma and adenoid cystic carcinomas are less clear-cut, and both have higher risks of distant dissemination, both at presentation and with follow-up.

Wedge biopsy (which may be video-assisted, ie video-assisted thoracic surgery (VATS)) is a type of lung-sparing surgery for distal lung masses. ${ }^{33}$ It is often used as an initial diagnostic procedure, and if carcinoma is found then a standard lobectomy is performed if the patient has sufficient pulmonary function. The use of VATS alone as an oncologic procedure is not well understood and controversial, as the long-term results of local control are not yet known. Evidence points to better survival and fewer recurrences in patients with lobectomies than lesser resections, ${ }^{34,35}$ although some studies have found no difference in survival. ${ }^{36}$ Our study did not investigate the adequacy of resection of wedge biopsies, but did examine the incidence of positive margins in completion lobectomy specimens who had undergone prior wedge biopsies. All patients who had had wedge biopsies with subsequent completion lobectomies had negative bronchial margins. Thus frozen sections were of no benefit in this group in our experience. Since we have shared this information with the members of the Division of Cardiothoracic Surgery, we no longer routinely receive requests for frozen section evaluation of bronchial margins in completion lobectomies. This saves the cost of a frozen section and operating room time for all such patients (approximately $\$ 150 /$ case and 15-20 min of OR time).

\section{Conclusions}

FSs of bronchial margins are particularly helpful for central tumors where taking additional tissue (sleeve resection or additional lobe) is feasible if initial margins are positive. Submucosal, peribronchial and lymphatic space invasion by tumor at the margin are more difficult to identify at FS than mucosal tumor. Gross evaluation of tumor distance from margins is problematic, and microscopic tumor often extends proximally from gross tumor masses. In our study $3 \mathrm{~cm}$ was the furthest distance microscopic tumor extended, and tumors grossly present greater than $3 \mathrm{~cm}$ from the margin did not require a frozen section. Completion lobectomies following wedge resections of peripheral tumors in which the wedge was felt to encompass the entire tumor did not benefit from frozen section of the bronchial margin in our series. Bronchial margins in all such cases were negative, whether a frozen section was performed or not. Bronchial salivary gland-type tumors have a higher incidence of positive margins than usual bronchogenic non-small-cell carcinoma, and frozen section is particularly helpful in these tumor types.

\section{References}

1 Cotton RE. The bronchial spread of lung cancer. Br J Dis Chest 1959;53:142-150.

2 Fang D, Zhang D, Huang G, et al. Results of surgical resection of patients with primary lung cancer: a retrospective analysis of 1,905 cases. Ann Thorac Surg 2001;72:1155-1159.

3 Gebitekin C, Gupta NK, Satur CM, et al. Fate of patients with residual tumour at the bronchial resection margin. Eur J Cardiothorac Surg 1994;8:339-342.

4 Ghiribelli C, Voltolini L, Paladini P, et al. Treatment and survival after lung resection for non-small cell lung cancer in patients with microscopic residual disease at the bronchial stump. Eur J Cardiothorac Surg 1999;16:555-559.

5 Hoffman HS, Taege C, Lautenschlager C, et al. Microscopic (R1) and macroscopic (R2) residual disease in patients with resected non-small cell lung cancer. Eur J Cardiothorac Surg 2002;21:606-610.

6 Kaiser LR, Fleshner P, Keller S, et al. Significance of extramucosal residual tumor at the bronchial resection margin. Ann Thorac Surg 1989;47:265-269.

7 Massard G, Doddoli C, Gasser B, et al. Prognostic implications of a positive bronchial resection margin. Eur J Cardiothorac Surg 2000;17:557-565.

8 Miller DL, McManus KG, Allen MS, et al. Results of surgical resection in patients with N2 non-small cell lung cancer. Ann Thorac Surg 1994;57:1095-1100.

9 Shields TW. The fate of patients after incomplete resection of bronchial carcinoma. Surg Gynecol Obstet 1974;139:569-572.

10 Snijder RJ, Brutel de la Riviere A, Elbers HJ, et al. Survival in resected stage I lung cancer with residual tumor at the bronchial resection margin. Ann Thorac Surg 1998;65:212-216.

11 Soorae AS, Stevenson HM. Survival with residual tumor on the bronchial margin after resectin for bronchogenic carcinoma. J Thorac Cardiovasc Surg 1979;78:175-180.

12 Wada H, Fukuse T, Hitomi S. Long-term survival of surgical cases of lung cancer. Lung Cancer 1995;13:269-274.

13 Wertzel H, Siebert H, Lange W, et al. Results after surgery in stage I bronchogenic carcinoma. Thorac Cardiovasc Surg 1998;26:365-369.

14 Hughes RK, Tildon TT. Prognosis of residual invasive cancer at the margin of bronchial resection. Ann Thorac Surg 1966;2:102-105.

15 Jeffery RM. Survival in bronchial carcinoma. Tumour remaining in the bronchial stump following resection. Ann R Coll Surg Engl 1972;51:55-59. 
16 Law MR, Hodson ME, Lennox. Implications of histologically reported residual tumour on the bronchial margin after resection for bronchial carcinoma. Thorax 1982;37:492-495.

17 Liewald F, Hatz RA, Dienemann H, et al. Importance of microscopic residual disease at the bronchial margin after resection for non-small cell carcinoma of the lung. J Thorac Cardiovasc Surg 1992;104:408-412.

18 Kara M, Sak SD, Orhan D, et al. Changing patterns of lung cancer; (3/4 in.) $1.9 \mathrm{~cm}$; still a safe length for bronchial resection margin? Lung Cancer 2000;30: 161-168.

19 Kayser K, Anyanwu E, Bauer HG, et al. Tumor presence at resection boundaries and lymph-node metastasis in bronchial carcinoma patients. Thorac Cardiovasc Surg 1993;41:308-311.

20 International Union Against cancer. In: Sobin LH, Wittekind Ch (eds). TNM Classification of Malignant Tumors. Wiley-Liss: New York, 1997, p 13.

21 Kara M, Dizbay Sak S, Orhan D, et al. Proximal bronchial extension with special reference to tumor localization in non-small cell lung cancer. Eur J Cardiothorac Surg 2001;20:350-355.

22 Griess DF, McDonald JR, Clagett OT. The proximal extension of carcinoma of the lung in the bronchial wall. J Thorac Surg 1945;14:362-368.

23 Passlick B, Sitar I, Sienel W, et al. Significance of lymphangiosis carcinomatosa at the bronchial resection margin in patients with non-small cell lung cancer. Ann Thorac Surg 2001;72:1160-1164.

$24 \mathrm{Lad} \mathrm{T}$. The comparison of CAP chemotherapy and radiotherapy to radiotherapy alone for resected lung cancer with positive margin or involved highest sampled paratracheal node (stage IIIA). LCSG 791. Chest 1994;106(Suppl):302-306S.

25 Yousem SA, Hochholqer L. Mucoepidermoid tumors of the lung. Cancer 1987;60:1346-1352.
26 Heitmiller RF, Mathisen DJ, Ferry JA, et al. Mucoepidermoid lung tumors. Ann Thorac Surg 1989;47: 394-399.

27 Moran CA. Primary salivary gland-type tumors of the lung. Semin Diagn Pathol 1995;12:106-122.

28 Moran CA, Suster S, Koss MN. Primary adenoid cystic carcinoma of the lung. A clinicopathologic and immunohistochemical study of 16 cases. Cancer 1994;73:1390-1397.

29 Conlan AA, Payne, WS, Woolner LB, et al. Adenoid cystic carcinoma (cylindroma) and mucoepidermoid carcinoma of the bronchus. Factors affecting survival. J Thorac Cardiovasc Surg 1978;76:369-377.

30 Vadasz P, Egervary M. Mucoepidermoid bronchial tumors: a review of 34 operated cases. Eur J Cardiothorac Surg 2000;17:566-569.

31 Suen HC, Meyers BF, Guthrie T, et al. Favorable results after sleeve lobectomy or bronchoplasy for bronchial malignancies. Ann Thorac Surg 1999;67: 1557-1562.

32 Ohta Y, Yachi $\mathrm{T}$, Oda $\mathrm{M}$, et al. Bronchial sleeve resection with complete preservation of the lung for carcinoma. Respiration 2001;68:528-532.

33 Kotoulas C, Lazopoulos G, Foroulis C, et al. Wedge resection of the bronchus: an alternative bronchoplastic technique for preservation of lung tissue. Eur J Cardiothorac Surg 2001;20:679-683.

34 Miller DL, Rowland CM, Deschamps C, et al. Surgical treatment of non-small cell lung cancer $1 \mathrm{~cm}$ of less in diameter. Ann Thorac Surg 2002;73: 1545-1551.

35 Van Schil PE. Surgery for non-small cell lung cancer. Lung Cancer 2001;34:S127-S132.

36 Thomas P, Doddoli C, Yena S, et al. VATS is an adequate oncological operation for stage I non-small cell lung cancer. Eur J Cardiothorac Surg 2002;21: 1094-1099. 